\title{
Development and clinical use of a computer assisted decision support system for the interpretation of alkaline phosphatase isoenzyme patterns
}

\author{
Entwicklung und klinischer Einsatz eines wissensbasierten Systems zur \\ Entscheidungsunterstützung für die Interpretation von Isoenzymmustern der \\ alkalischen Phosphatase
}

V.O. Van Hoof', L.G. Lepoutre', M.E. De Broe 2

'Department of Clinical Chemistry

2Department of Nephrology/Hypertension, University Hospital Antwerp, Belgium

\begin{abstract}
Summary:
A computer-assisted decision support system for the clinical interpretation of alkaline phosphatase isoenzyme patterns (CAIAPP) was built to increase the diagnostic potential of this laboratory test. Alkaline phosphatase (ALP, EC 3.1.3.1.) is a plasma-membranebound ecto-enzyme that can be resolved into tissue non-specific (liver/bone), placental, germ-cell and intestinal ALP. Each of these isoenzymes can be separated into several isoforms which, based on their structural characteristics, were identified as soluble dimeric (Sol), anchor-bearing (Anch) and membrane-bound (Mem) ALP. The reference ranges implemented in the CAIAPP system are the 5th and the 95th percentile of each isoenzyme/isoform, per age group and gender, determined in apparently healthy individuals ( $n=1383 ; 4-65$ years old). Changes in the serum ALP isoenzyme pattern can be related to specific disease states, especially bone and liver disease. To deal with aspecific changes of the isoenzyme pattern however, we had to implement a "fuzzy" zone between the values obtained for the healthy reference population and those obtained for a background population of patients without liver or bone disease $(n=101)$. The knowledge base (KB) was further extended with the results of several clinical studies, e.g. the predictive values for Sol- and Anch-bone ALP, Sol-liver and/or Memliver ALP for the diagnosis of bone and liver metastasis in patients with malignant disease. The actual $K B$ hat 443 rules, divided over 5 main modules: normal pattern, bone diseases, liver diseases, intestinal ALP and placental ALP. The CAIAPP system is unidirectionally linked to the central laboratory computer. Input data are restricted to those immediately available in the biochemistry laboratory. The system provides the clinician with a report that gives a clinical interpretation of the ALP isoenzyme pattern which takes into account the results of other laboratory tests and eventually suggests which additional tests are needed. Since its start in 1989, > 8000 ALP isoenzyme patterns have been routinely evaluated. A clinical evaluation was performed in $1990(n=363)$. A complete or partial agreement of the suggestions made by the expert system and the patient's records was obtained in $82 \%$ and $5 \%$ of the cases respectively. The clinical relevance of the CAIAPP system is continuously re-evaluated for further refinement of the rules.
\end{abstract}

\section{Keywords:}

Alkaline phosphatase isoenzyme patterns - knowledge-based systems - evaluation of knowledge-based systems-Pro.M.D.

\section{Zusammenfassung:}

Es wurde ein wissensbasiertes System zur Entscheidungsunterstützung für die klinische Interpretation der Isoenzymmuster der alkalischen Phosphatase (AP) entwickelt (CAIAPP-System), um die diagnostischen Möglichkeiten der klinischen Interpretation dieses Testverfahrens zu erhöhen. Alkalischè Phosphatase (ALP, EC 3.1.3.1.) ist ein Plasmamembran gebundenes Ekto-Enzym, welches in nicht gewebespezifische (Leber, Knochen), Plazenta-, Keimzell- oder intestinale Isoenzymformen unterteilt werden 
kann. Jedes dieser Isoenzyme kann weiter in verschiedene Isoformen gruppiert werden, die basierend auf ihren strukturellen Eigenschaften in lösliche Dimere (SOL), Anker-tragende (ANCH) und Membrangebundene (MEM) Formen eingeteilt werden. Die Referenzwerte, die für jedes Isoenzym im CAIAPP-System implementiert wurden, entsprechen der fünften und fünfundneuzigsten Perzentile. Die Referenzwerte wurden an offensichtlich gesunden Probanden $(n=1383)$ im Alter von 4 bis 65 Jahren unterteilt nach verschiedenen Altersgruppen und Geschlecht erhoben. Die Veränderungen des AP-Isoenzymmusters können verschiedene Krankheitsstadien, insbesondere Knochenund Lebererkrankungen zugeordnet werden. Um unspezifische Änderungen im Enzymmuster zu erkennen, mußten wir eine "Fuzzy"-Zone zwischen den Werten der gesunden Referenz-Population und den erhöhten AP-Werten von Patienten ohne Leber-oder Knochenerkrankung ( $n=101$ ) einrichten. Im Zusammenhang mit der Diagnostik von Knochen- und Lebermetastasen bei Patienten mit maligner Erkrankung wurde die Wissensbasis mit Ergebnissen verschiedener klinischer Studien erweitert, so z. B. mit den prediktiven Werten für SOL- und ANCH-Knochenisoenzymformen sowie für SOLLeber- und/oder MEM-Leberisoenzymformen. Die aktuelle Wissensbasis hat $443 R e-$ geln, die auf 5 Hauptmodule aufgeteilt sind: Normales Muster, Knochenerkrankung, Lebererkrankung, intestinale AP und Plazenta-AP. Das CAIAPP-System ist unidirektional mit dem zentralen Computer verbunden. Die Eingabedaten sind auf direkt im biochemischen Labor verfügbare Werte beschränkt. Das System stellt dem Kliniker eine Befundbeschreibung zur Verfügung, in welcher die klinische Interpretation der APIsoenzymmuster mit Berücksichtigung anderer Laborresultate enthalten ist und schlägt eventuell notwendige weitere Untersuchungen vor. Seit 1989 sind über 8000 APIsoenzymmuster im Routinebetrieb evaluiert worden. 1990 erfolgte eine klinische Evaluation an 363 Patienten. Eine vollständige oder teilweise Übereinstimmung der Vorschläge des Befundungssystems mit den Krankenblätter der Patienten wurden in $82 \%$ der Fälle gefunden. Bei $5 \%$ der Fälle fand sich eine teilweise Übereinstimmung. Die klinische Nutzung des CAIPP-Systems wird kontinuierlich weiterbewertet, um weitere Verbesserungen der Regeln durchzuführen.

\section{Schlüsselwörter:}

Alkalische Phosphatase-Isoenzyme - wissensbasierte Systeme - Evaluation wissensbasierter Systeme - Pro.M.D.

\section{Introduction}

Alkaline phosphatase (ALP,EC 3.1.3.1.) is a metalloenzyme consisting of a group of isoenzymes, all glycoproteins, encoded for by at least four different gene loci: tissue non-specific, placental, germ-cell and intestinal ALP. Bone and liver ALP are formed through pos-translational modifications (differential glycosylation) of the tissuenon-specific gene product $(1-4)$.

ALPs are widely distributed in nature and are present in virtually all species, from bacteria to man, with a high degree of homology, an indication that the enzyme is involved in fundamental biochemical processes. Its exact physiological role however is as yet unknown. The abundance of ALP on membranes dealing with the active transport of many substances suggests a possible role of ALP in this transport; e.g. intestinal ALP on the membranes of duodenal cells and small intestinal enterocytes might be involved in the transport of lipids (4). Bone ALP activity is very high in osteoblasts, and in 1923 Robison first recognized the important role of ALP in the mineralization process (5). The strongest evidence that ALP is involved in bone formation was given by the clinical presentation of congenital hypophosphatasemia, a rare disorder in which tissue non-specific ALP is lower or even absent (6). The very severe forms of this disease are not compatible with life, but the milder forms are characterized by mineralization defects and dental problems, which may even be delayed till adulthood.
ALP is a membrane-bound ectoenzyme, linked to the plasma membrane by means of a phosphatidylinositol glycan anchor (7). Placental, germ-cell, intestinal and the tissue non-specific ALPs consist of several isoforms which, based on their structural characteristics, were identified as soluble dimeric (Sol-) ALP, and two hydrophobic forms: anchor-bearing (Anch-) and membrane-bound (Mem-) ALP (8) (Figure 1). In normal human serum, the major isoenzymes are bone and liver ALP, which mainly circulate in their soluble dimeric form.

\section{Separation of the ALP isoenzymes and isoforms}

As several isoenzymes and isoforms may contribute to a rise of total ALP activity, separation of ALP is likely to enhance the diagnostic potential of the test. Many different methods have been described which serve this purpose. Heat denaturation (9) and specific inhibition by amino acids $(10)$ are among the eldest. Recently, methods using either lectin precipitation (11) or a monoclonal anti-body (12) to determine the bone ALP have become available. Advantages of these methods are that they are quick and can be run on chemistry automates. Serious disadvantages are that they do not give any information regarding the other isoenzymes, and that they show considerable cross-reactivity with liver ALP. Electrophoretic separation on the other hand offers an overview of all the isoenzymes present. Nowadays most commercially available electrophoretic systems are easy-to-use, reproducible and sensitive (13). However, as none of the available electrophoretic methods can completely resolve all the ALP iso- 


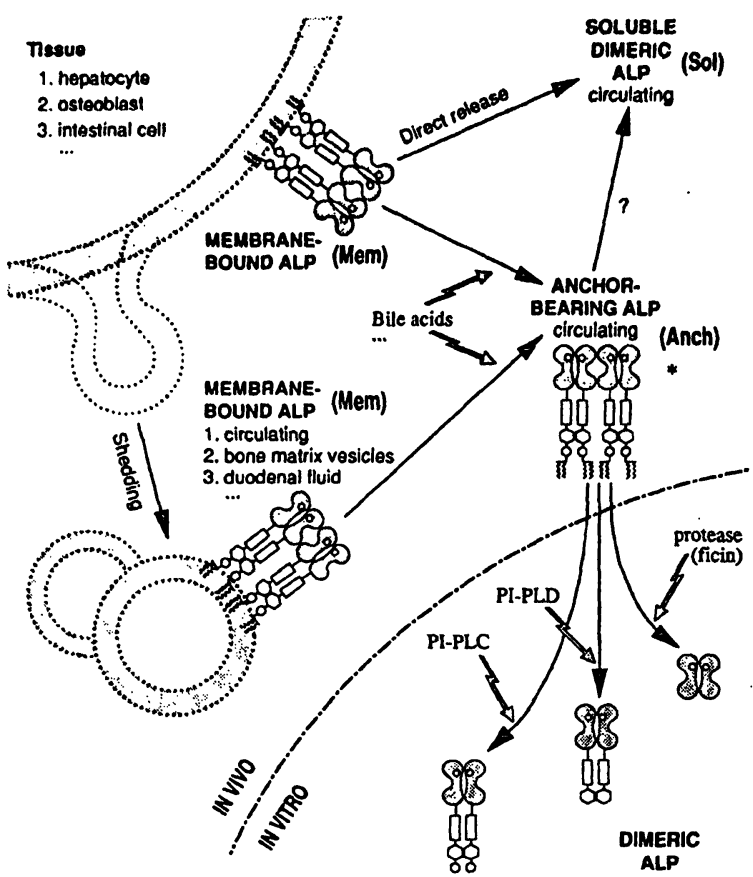

Fig. 1: Schematic representation of the various forms of ALP that are present in vivo and their conversions obtained by in vitro experiments (according to ref. 8, with permission).

*: this drawing represents only one of the several structures of anchorbearing ALP. The results of gel chromatographic experiments without detergents suggest that more complex structures involving more than two dimers (probably held together by interactions between their hydrophobic tails), as well as anchor-bearing dimers can circulate in human serum too.

Sol- = soluble dimeric liver/bone/intestinal/placental ALP

Anch- = anchor-bearing liver/bone/intestinal/placental ALP ("variant" forms)

Mem- = membrane-bound liver/bone/intestinal ALP ("high-Mr" forms) $P I-P L C=$ phosphatidylinositol specific phospholipase- $C$

$P I-P L D=$ phosphatidylinositol specific phospholipase-D

enzymes, it is often necessary to perform one or more sample treatments to identifiy the fractions correctly, which is particularly true for complex isoenzyme patterns. One of these treatments consists in the addition of a polyclonal or monoclonal antibody against one of the ALP isoenzymes, which results in an altered mobility of the bound isoenzyme. In this way a 5 min incubation with a polyclonal anti-placental antibody, cross-reacting with intestinal ALP, allows for a correct quantification of Solbone ALP in the Isopal system when Anch-intestinal ALP or Sol-placental ALP are present (both fractions comigrate with Sol-bone ALP with the Beckman system). One can also treat the samples with neuraminidase which reacts with sialic acid residues and thus alters the electrophoretic mobility of the sialilated isoenzymes (all but intestinal ALP) (14). In this way, a 30 min incubation with neuraminidase results is a better separation of bone and liver ALP in the Isopal system, only needed when bone ALP activity exceeds $50 \%$ of the total ALP activity (Figure 2).

\section{Reference ranges}

Using the Isopal agarose electrophoretic system (Beckman, Brea, CA) (15), reference ranges for the different

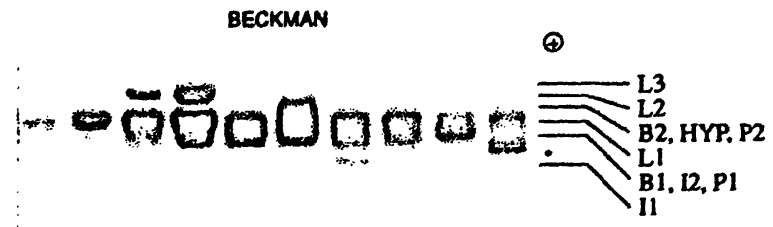

$\begin{array}{lllllllllll}1 & 2 & 3 & 4 & 5 & 6 & 7 & 8 & 9 & 10 & \odot\end{array}$
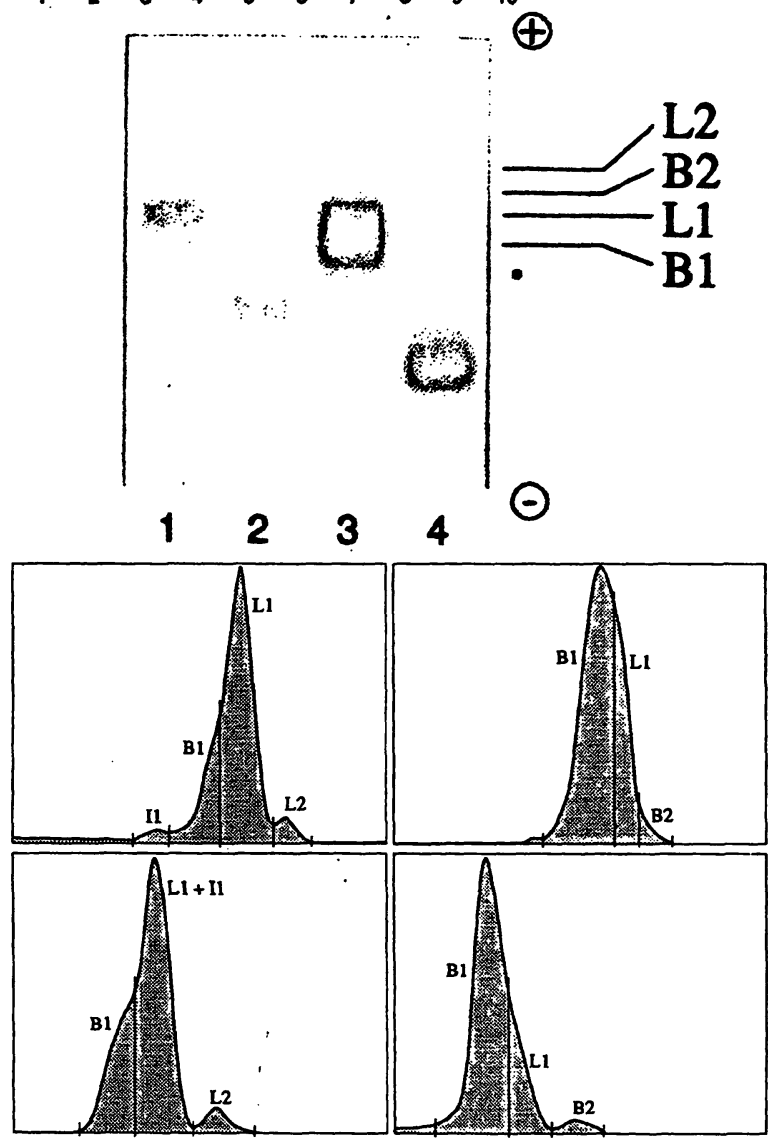

Figure 2:

a. Typical ALP isoenzyme patterns obtained with the Beckman system. Lanes: 1 = normal pattern; $2=$ high Sol-liver $A L P+$ trace of Mem-liver ALP; $3=$ high Sol-liver +Mem-liver ALP; $4=$ high Sol-liver + Mem-liver ALP+ lipoprotein-bound liver ALP; 5 = high Sol-bone + Anch-bone ALP; $6=$ transient hyperphosphatasemia; $7=$ high Sol-intestinal ALP + trace of Anch-intestinal $A L P ; 8=$ sample from lane 7 treated with polyclonal antiintestinal antiserum; 9 = high Sol-placental ALP and Anch-placental ALP; 10 = sample from lane 9 incubated with monoclonal anti-placental antiserum. Note: the symbols indicate ALPfractions of untreated samples. L1 = Sol-liver ALP; L2 = Mem-liver ALP ("high-Mr"); $L 3=$ lipoproteinbound liver ALP ("ultra-fast"); $11=$ Sol-intestinal ALP; 12 and $13=$ Anchintestinal ALP ("intestinal variant"); HYP = fraction of transient hyperphosphatasemia; $B 1$ = Sol-bone ALP; B2 = Anch-bone ALP l"bone variant"); $P 1=$ Sol-placental ALP; P2 = Anch-placental ALP ("placental variant").'. (According to ref 13 , with permission).

b. Treatment with neuraminidase. Lanes: $1=$ serum with low Sol-bone +Anch-boneALPactivity;2=serum fromlane 1 treated withneuraminidase for 30 min; 3=serum with high Sol-boneALPandAnch-boneALPactivity; $4=$ serum from lane 3 treated with neuraminidase for $30 \mathrm{~min}$.

c. Densitometric scannings of gel $b$. Top left: lane 1 and bottom left: lane 2; top right: lane 3 and bottom right: lane 4. 
ALP isoenzymes and isoforms were established in 1383 healthy individuals ( $4-65$ years old) (16). The ALP isoenzyme pattern varies according to age and gender. Solliver ALP activity is quite stable throughout life, while Solbone ALP activity is much higher in children and adolescents as compared to adults. Sol-bone ALP activity reaches a maximum in adolescence, approximately two years earlier in girls $(9-11$ years) than boys ( $13-14$ years), in whom higher values are found. Thereafter, Sol-bone ALP activity declines steeply and adult values are reached at the age of $15-17$ in girls and the age of $19-20$ in boys. Low activities of anch-bone ALP (formerly called "bone variant $\left.A L P^{\prime \prime}\right)$ are found in the serum of $99 \%$ of the healthy children. This fraction disappears when adult values of Solbone ALP are reached. Mem-liver ALP ("fast liver ALP", "High-Mr ALP", "koinozyme") consists of vesicular structures, formed by "shedding" of the liver cell membrane (17). This fraction is characteristically found in cholestasis and is normally not present in children. Trace amounts of Mem-liver ALP can be found in healthy adults. Intestinal ALP activity rises following the ingestion of a fatty meal (18) and the presence of intestinal ALP is more frequent in individuals with blood groups B or 0 who are secretors (19). Depending on the method used Anch-intestinal ALP activity can be demonstrated in about one to two thirds of the reference samples. Low activities of placental ALP are present in serum from healthy, non-pregnant individuals, but they can only be detected by very sensitive immunological procedures (20).

\section{ALP isoenzyme patterns in disease}

Several pathological factors influence the cellular expression and liberation of ALP isoenzymes in the blood. Within the scope of this paper, it is impossible to give credit to all the workers who have studied ALP in disease. For a more extended version and a more complete list of references we refer to our recent review article (8).

The clinical significance of the isoenzyme patterns can be quite different in children and adolescents as compared to adults, therefore both groups will be considered separateley.

\section{Children and adolescents}

A high osteoblastic activity results in an increase of bone ALP activity. Rickets and healing fractures are common causes of an increased osteoblastic activity in children. Rickets occurs in a variety of disorders, such as vitamin $D$ deficiency and conditions with an impaired proximal tubular function, and is characterized by a defective mineralization of the bone. Less frequent causes of a high bone ALP activity in children are osteoid osteoma, primary osteogenic sarcoma and secundary tumors of the bone. The aforementioned bone diseases in children all have to be differentiated from a benign and rather common condition called "transient hyperphosphatasemia of childhood". This phenomenon is more common in young children ( 0 - 5 years old) and is characterized by the transient appearance of a particular fraction of unknown origin (showing features of both bone and liver ALP), which is often accompanied by a prominent rise of bone ALP activity. Transient hyperphosphatasemia can be readily diagnosed by electrophoresis, thus avoiding tedious and sometimes invasive investigations.

Low bone ALP activity reflects a low osteoblastic activity and can be found in growth hormone deficiency, idiopatic hypoparathyroidism and congential hypophosphatasemia. A temporary depression of bone ALP activity can be observed in severly ill children and is often followed by a "catch-up" increase during convalescence. Determination of the ALP isoenzyme pattern is particularly useful in children with liver diseases. Total ALP activity may be well within the broad reference ranges for children, while Solliver ALP is raised and/or Mem-liver ALP is present. The presence of Mem-liver ALP in infants can be due to congential bile duct atresia or a bile duct cyst, but is also seen in septicemia. In children and adolscents a similar isoenzyme pattern is present in primary sclerosing cholangitis and in cases of cystic fibrosis with cholestatic liver involvement. Drug induced and viral hepatitis in children cause a rise of Sol-liver ALP activity, often accompanied by a Mem-liver ALP fraction, depending on the degree of cholestasis. Following a succesful treatment of these conditions, the isoenzyme pattern quickly returns to normal. Though rare in childhood, a rise of Sol-liver ALP and the appearance of Mem-liver ALP can be due to liver metastasis of a malignant tumor. The presence of a raised intestinal type ALP activity will only very rarely indicate intestinal problems (e.g. necrotizing colitis with colon perforation in newborns). Diseases of the colon and small intestine will rather lead to changes in the tissue nonspecific isoenzymes e.g. a rise in bone ALP activity due to impaired absorption of vitamin D. Finally, benign inherited forms of hyperphosphatasemia have been described for tissue non-specific ALP, intestinal and placental ALP.

\section{Adults}

Common causes of high osteoblastic activity and an increase in bone ALP activity in adults are Paget's disease of bone, the healing of a fracture, osteomalacia (the equivalent of rickets in children) and osteoblastic metastasis. Less common causes are primary hyperparathyroidism, myositis ossificans, osteosclerosis and primary osteosarcoma. Low bone ALP activity is found in conditions with low osteoblastic activity such as vitamin B12 deficiency, multiple myeloma (approximately $80 \%$ of the patients) and hypoparathyroidism. Conflicting reports have been published concerning bone ALP activity in osteoporosis: low, high and normal activities have been described. Many drugs have an influence on bone ALP activity, such as antiepileptic drugs (rise of ALP activity, mostly of bone type), cyclosporin (rise of bone ALP activity), fluoride (rise of bone ALP activity, but high concentrations inhibit bone ALP) and vitamin D (decline of bone ALP activity).

Sol-liver ALP is the largest ALP component of normal human serum. Liver diseases such as acute and chronic hepatitis, cirrhosis, primary or metastatic carcinoma of the liver and acute or chronic biliary obstruction are associated with an increase of Sol-liver and/or Mem-liver ALP activity, depending on the (accompanying) degree of cholestasis. Mem-liver ALP bearing vesicles are released from the liver plasma membrane through the detergent action of bile. It is a good marker of cholestasis which, however, does not differentiate the extra-from the intrahepatic forms, neither does it differentiate malignant from benign liver disease nor primary from secundary liver tumors. In severe cholestatic processes, either extra-hepatic or generalized intra-hepatic, ALP can associate with lipoproteins (e.g. lipoprotein-X). This results in a liver-ALP expressing structure which is clearly different 
from the membrane-bound form and has been called "ultra-fast ALP". One of the most characteristic features of the ALP isoenzyme pattern in liver cirrhosis is the presence of elevated activities of Sol-intestinal ALP. In normal circumstances intestinal ALP, being an asialoglycoprotein, is cleared by the hepatic asialoglycoprotein receptor. Deficiency of this receptor in cirrhosis is probably the cause of high Sol-intestinal ALP activities in this condition.

Cut-off values for liver and bone type ALP for the detection of metastasis were established by means of ROC-curves. The positive predictive value of the combination of Soland Mem-liver ALP was definitively higher thant that of total ALP for the detection of liver metastasis $170 \%$ versus $53 \%)$. Its negative predictive value on the other hand was almost equal to that of total ALP ( $89 \%$ versus $91 \%)$. The positive predictive value of bone ALP in the detection of bone metastasis was much higher that that of total ALP $(74 \%$ versus $49 \%)$, while its negative predictive value was lower $(84 \%$ versus $81 \%)$. Since bone ALP is a specific marker of osteoblastic activity it will not rise in osteoclastic metastasis.

A broad variety of ALP isoenzyme patterns is present in chronic renal failure. Hemochromatosis, hepatitis $B$, hepatitis $C$ or other viral infections such as CMV are the most common causes of liver disease and a rise of Solliver and/or Mem-liver ALP activity in patients with end stage renal failure treated with hemodialysis. Furthermore there is a high incidence of raised activities of intestinal type ALP in patients with advanced chronic renal failure. A possible cause in the decreased potential of the liver to clear this asialoglycoprotein. An other mechanism could be a competition of intestinal ALP with other asialoproteins, know to be raised in chronic renal failure. Bone ALP activity can either be raised (secundary hyperparathyroidism) or depressed (adynamic bone disease) in chronic renal failure. Adynamic bone disease is characterized by a reduced osteoblast surface and low bone formation rate. Originally this condition was considered to be due to aluminium overload. It has been shown however that other possible causes for adynamic bone disease in chronically dialyzed patients are parathyroidectomy, excessive parathyroid suppression with vitamin $D$ an a high calcium load. Intestinal type ALP in urine is a specific and sensitive marker for alterations of the S3 segment of the proximal tubule, a part of the kidney that is particularly vulnerable for several nephrotoxins.

As was discussed in a previous paragraph, ALP can form complexes with other serum components, such as lipoproteins, but it can also be complexed to immunoglobulins. Due to their peculiar migration pattern, these complexes can be readily diagnosed by electrophoresis. The incidence of ALP-immunoglobulin complexes in a healthy population is about $0.03 \%(21)$, and there is an increased incidence in elderly subjects and in diseased populations. The incidence of immunoglobulin-bound complexes has been reported to be higher in patients with ulcerative colitis, but no consistent pattern of disease association has emerged yet. Some ALP isoenzymes can be reexpressed in malignant disease. In this way, placental ALP ("Regan" isoenzyme) has been reported to be reexpressed in malignancies of the lung, the gastro-intestinal tract, the uterus and other tissues, but only in a proportion of the patients with these malignancies. It is however a useful marker in the detection and follow-up of carcinoma of the ovary. Germ-cell ALP activity ("Nagao" isoenzyme) is elevated in patients with germ cell tumors and in choriocarcinoma cell lines. It has been reported in a variety of other tumors but it is almost invariably produced by seminomas. Elevated levels have been found in smokers. The embryonic gene for foetal intestinal ALP can also be reexpressed by cancer cells ("Kasahara" isoenzyme). It has been mainly found in hepatoma, but is occasionally produced by other tumors too.

\section{CAIAPP}

The previous paragraphs clearly demonstrate that separating ALP into its isoenzymes and isoforms adds considerable value to the mere measurement of total ALP activity. However, the routine performance of this test will only enhance the quality of the information offered to the clinician when it is correctly interpreted. This implies the need for proper training of the technicians to correctly identify and quantify the isoenzyme fractions, but also the need for a considerable knowledge on the clinical significance of the isoenzyme patterns. To fulfil the latter need, we have built a computer assisted decision support system for the clinical interpretation of ALP isoenzyme patterns (CAIAPP) (22), with help of the ProM.D. expert system shell. Pro-M.D. is a rule-based shell, written in Prolog with a data-driven (forward-chaining) approach. It was specifically designed by Trendelenburg and Pohl to interprete laboratory results $(23,24)$. The actual knowledge base (KB) has 443 rules, divided over 5 main modules: normal pattern, bone diseases, liver diseases, intestinal and placental ALP. An example of a
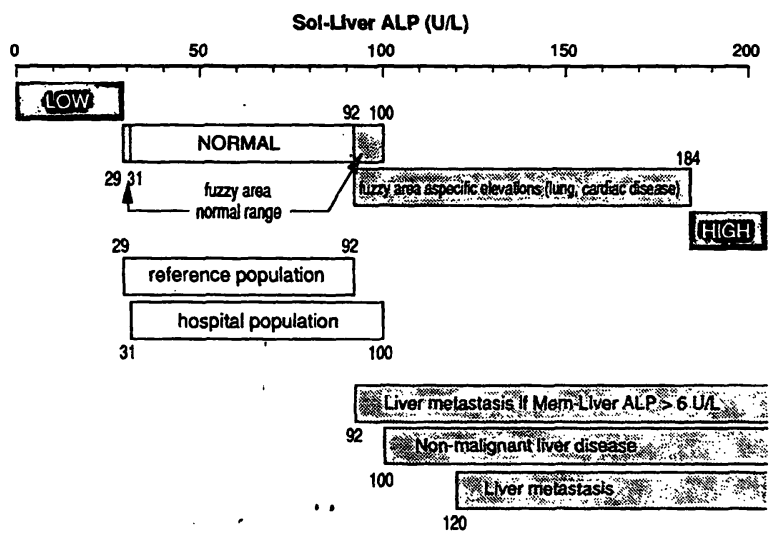

Fig. 3: Example of a set of rules in the module "Placental ALP": if $P L A P>0$ then go to PLAP_set

module PLAP_set

if SEX IS FEMALE and $14>$ AGE $=<45$ then PREGNANT

if SEX IS MALE and AGE $=<40$ then PLAMP1

result PREGNANT means:

IN A WOMAN OF CHILDBEARING AGE THE PRESENCE OF PLACENTAL ALP:

- MOST PROBABLY INDICATES PREGNANCY

- IN EXCEPTIONAL CASES THE PRESENCE OF AN OVARIAN TUMOR.

result PLAMP1 means:

THE PRESENCE OF PLACENTAL ALP IN A MAN OF THIS AGE SUGGESTS:

- THE PRESENCE OF A TESTICULAR TUMOR

- IN EXCEPTIONAL CASES THE PRESENCE OF OTHER TUMORS LIKE BRONCHIAL CARCINOMA. 
set of rules is shown in Figure 3. The CAIAPP system is unidirectionally linked to the central laboratory computer and input data are restricted to those immediately available in the biochemistry laboratory. The system provides the clinician with a report that lists and explains possible diagnoses, taking into account the results of other laboratory tests and eventually suggesting which additional tests are needed. The following data are implemented: age, gender, serum total ALP activity, ALP isoenzymes, ASAT, ALAT, total bilirubin, creatinin and calcium. The implemented reference ranges are the 5 th and the 95th percentile of each isoenzyme/isoform per age group and gender, determined in the reference population that was described in a previous paragraph. To deal with aspecific changes of the isoenzyme pattern, we had to implement a "fuzzy" zone between the values obtained for the healthy reference population and those obtained for a background hospital population of 101 patients whose files contained no evidence of liver or bone disease, or of any of the other conditions known to influence the isoenzyme pattern. As can be seen in Figure 4, this "fuzzy" zone is broader for bone ALP (both lower and higher values were found in the hospital population) than for Sol-liver ALP, where there was a shift towards higher values only. An additional "fuzzy" zone was necessary for Sol-and Mem-liver ALP to deal with the changes in the isoenzyme pattern observed in cardiovascular diseases and after lung embolism. These changes are probably due to congestive hepatomegaly, provoked by acute failure of the right ventricle and pulmonary hypertension, and thus they do not indicate the presence of "true" liver disease. We started with the first prototype of the CAIAPP system in october 1989 and by now, $>8000$ ALP isoenzyme patterns have been routinely evaluated. After proper testing, a clinical evaluation was performed for 363 patients in 1990. A complete or partial agreement

Sol-Bone ALP (Un)

Fig. 4: Schematic representation of the way cut-off values for bone and liver ALP were implemented in the CAIAPP system.

between the suggestions made by the expert system and the patient's records was obtained in $82 \%$ and $5 \%$ of the cases respectively. Since, the clinical relevance of the CAIAPP system has been.continuously re-evaluated, essentially through communication with the clinicians and review of the literature. This has lead to further refinement of the rules. A new clinical evaluation is in progress.

\section{References:}

1. Weiss, M.J.; Henthorn, P.S.; Lafferty, M.A.; Slaughter, C.; Raduch, M.; Harris, H. (1986): Isolation and characterization of cDNA encoding a human liver/bone/ kidney-type alkaline phosphatase. Proc. Natl. Acad. Sci. USA; 83: 7182-7186

2. Millan, J.L. (1986): Molecular cloning and sequence analysis of human placental alkaline phosphatase, J. Biol. Chem. 261: 3112-3115

3. Berger, J.; Garattini, E.;Hua, J.-C.; Udenfriend, S. (1987): Cloning and sequencing of human intestinal alkaline phosphatase cDNA. Proc. Natl. Acad. Sci. USA; 84: 695-698

4. Keiding, N.R. (1964): The alkaline phosphatase fractions of human lymph. Clin. Sci. 26: 291-297

5. Robison, R. (1923): The possible significance of hexosophosphoric esters in ossification in vitro. Biochem. J. 17: 286-293

6. Weiss, M.J.; Ray, K.; Fallon, M.D.; Whyte, M.P.; Fedde, K.N.; Lafferty, M.A.; Mulivor, R.A.; Harris, H. (1989): Analysis of liver/bone/kidney alkaline phosphatase mRNA, DNA, and enzymatic activity in cultured skin fibroblasts from 14 unrelated patients with severe hypophosphatasia. Am. J. Hum. Genet. 44: 686-694

7. Chakrabartty, A.; Stinson, R.A. (1985): Tetrameric alkaline phosphatase in human liver plasma membranes. Biochem. Biophys. Res. Commun. 131:328-335 8. Van Hoof, V.O.; De Broe, M.E. (1994): Interpretation and clinical significance of alkaline phosphatase isoenzyme patterns. Crit. Rev. Clin. Lab. Scie. 31: 197-293 (review)

9. Whitby, L.G.; Moss, D.W. (1975): Analysis of heat inactivation curves of alkaline phosphatase isoenzymes in serum. Clin. Chim. Acta 59: 361-367

10. Price, C.P. (1993): Multiple forms of human serum alkaline phosphatase: detection and quantitation. Ann. Clin. Biochem. 30: 355-372 (review)

11. Rosalki, S.B.;Foo, A.Y.;Burlina, A.;Prellwitz,W.;Stieber,P.;neumeier,D.; Klein, G.:Poppe, A.; Bodenmüller, H. (1993): Multicenter evaluation of Iso-ALP test kit for measurement of bone alkaline phosphatase activity in serum and plasma. Clin. Chem. 39: 648-652

12. Panigrahi, K.; Delmas, P.; Singer, F.; Ryan, W.; Reiss, O.; Fisher, R.; Miller, P.D. Mizrami, I.; Kress, B.C.; Christenson, R.H. (1994): Characteristics of a two-site immunoradiometric assay for human sleketal alkaline phosphatase in Serum. Clin. Chem. 40: 822-828

13. Van Hoof, V.O.; Van Mullem, M.; De Broe, M.E.; Lepoutre, L.G. (1993): Comparison of two commercially available systems for the electrophoretic separation of alkaline phosphatase isoenzymes. J. Chromatogr. 646: 235-243

14. Moss, D.W. Edwards, R.K. (1984): improved electrophoretic resolution of bone and liveralkaline phosphatase resulting from partial digestion with neuraminidase. Clin. Chim. Acta 143: 177-182

15. Van Hoof, V.O.; Lepoutre, L.G.; Hoylaerts, M.F.; Chevigné, R.; De Broe, M.E. (1988): Improved agarose electrophoretic method for separating alkaline phosphatase isoenzymes in serum. Clin. Chem. 34: 1857-1862

16. Van Hoof, V.O.; Hoylaerts, M.F.; Geryl; H.; Van Mullem, M.; Lepoutre, L.G.; De Broe, M.E. (1990):Age and sex distribution of alkaline phosphatase isoenzymes by agarose electrophoresis. Clin. Chem 36: 875-878

17. De Broe, M.E.; Roels, F.; Nouwen, E.J.; Wieme, R.J. (1985): Liver plasma membrane: the source of serum high molecular weight alkaline phosphatase in human serum. Hepatology 5: 118-128

18. Langman, M.J.S.; Leuthold, E.; Robson, E.B.; Harris, J.; Luffman J.E.; Harris, H. (1966): Influence of diet on the intestinal component of serum alkaline phosphatase in people of different ABO blood groups and secretor status. Nature 212: 41-43 19. Bamford, K.F.; Harris, H.; Luffman, J.E.; Robson, E.B.; Cleghorm, T.E. (1965): Serum-alkaline-phosphatase and the ABO blood-groups. Lancet 1: 530-531 20. Hendrix, P.G.; Hoylaerts, M.F.; Nouwen, E.J.; De Broe, M.E. (1990): Enzyme immunoassay of human placental and germ-cell alkaline phosphatase in serum. Clin. Chem. 36: 1793-1799

21. Hattori, Y.; Yamamoto, K.; Urabe, C.; Furuya, M.; Eguchi, H.; Hattori, H.; Miyaji, $\mathrm{T}$. (1985): Frequency of alkaline phosphatase-immunoglobulin complex among diseased and healthy populations. Clin. Chim. Acta 147: 155-158

22. Van Hoof, V.O.; Verpooten, G.A.; Lepoutre, L.G., De Broe, M.E. (1990): A computer assisted decision support system for the interpretation of alkaline phosphatase isoenzyme patterns. Clin. Chem. 36: 1014 (abstract)

23. Pohl, B.; Trendelenburg, C. (1988): Pro-M.D., a diagnostic expert system shell for clinical chemistry test result interpretation. Methods inf. Med. 27: 111-117

24. Trendelenburg, C.; Pohl, B. (1988): Pro.M.D., medizinische Diagnostik mit Expertensystemen. Georg Thieme Verlag, Stuttgart, ISBN 3-13-730301-X

\section{Anschrift des Verfassers:}

Viviane Van Hoof, MD, PhD

Dept. of Clinical Chemistry

University Hospital Antwerp

Wilrijkstraat 10

B-2650 Edegem 